\title{
Counterexamples to the sonic criterion
}

\author{
Volker Elling
}

\begin{abstract}
We consider self-similar (pseudo-steady) shock reflection at an oblique wall. There are three parameters: wall corner angle, Mach number, angle of incident shock. Ever since Ernst Mach discovered the irregular reflection named after him, it has been an open problem to predict precisely for what parameters the reflection is regular. Three conflicting proposals, the detachment, sonic and von Neumann criteria, have been studied extensively without a clear result.

We demonstrate that the sonic criterion is not correct. We consider polytropic potential flow and prove that there is an open nonempty set of parameters that admit a global regular reflection with a reflected shock that is transonic.

We also provide a clear physical reason: the flow type (sub- or supersonic) is not decisive; instead the reflected shock type (weak or strong) determines whether structural perturbations decay towards the reflection point.
\end{abstract}

\section{Introduction}

\subsection{The transition problem in shock reflection}

Reflection of an incident shock from a solid wedge is a classical problem of gas dynamics. It has been studied extensively by Ernst Mach 21, 18 and John von Neumann [25], among others.

Most commonly, reflection is studied in steady inviscid polytropi 11 compressible flow, for example when shocks in a nozzle are reflected from the walls. The reflections can be classified roughly into regular and irregular reflections; see [1] or [2, Figure 1] for a more detailed discussion. In either type, an incident shock impinges on a solid surface. In regular reflection (RR), the incident shock reaches a reflection point on the surface, continuing as a reflected shock (see Figure 1 top left).

\footnotetext{
${ }^{1}$ equation of state $p=(\gamma-1) \rho e, e$ internal energy per mass, $\gamma \in(1, \infty)$
} 


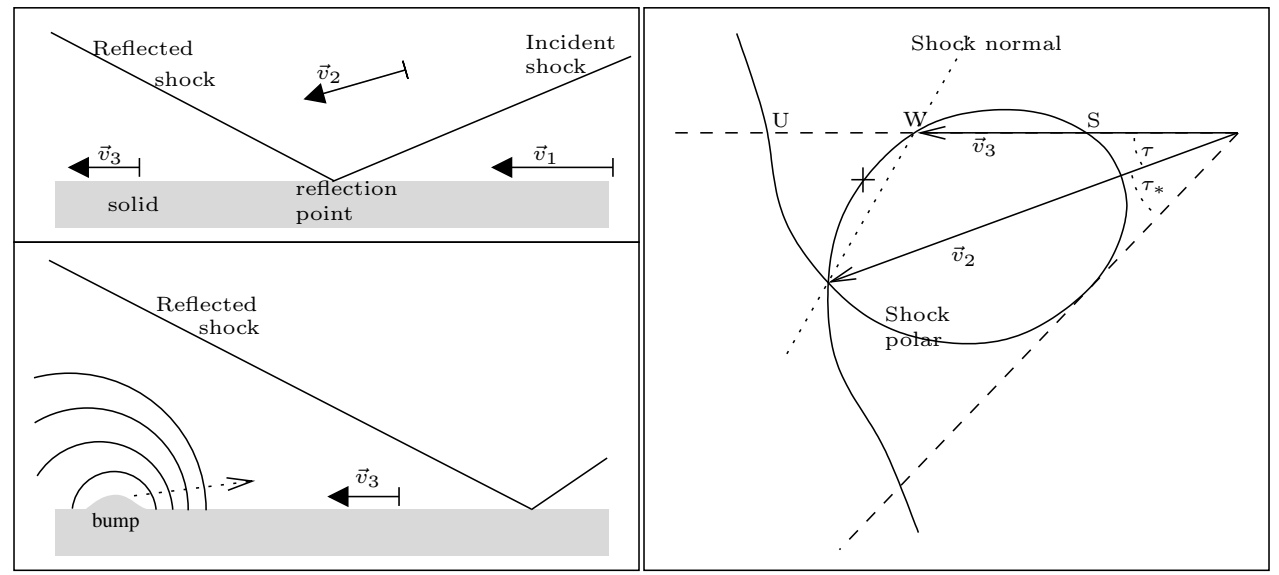

Figure 1: Left top: local RR. Left bottom: subsonic case. Right: fixed $\vec{v}_{2}$; each steady shock produces one $\vec{v}_{3}$ on the curve (shock polar, symmetric across $\vec{v}_{2}$; shock normal $\left.\| \vec{v}_{2}-\vec{v}_{3}\right)$. For $|\tau|<\tau_{*}$, three shocks satisfy $\tau=\measuredangle\left(\vec{v}_{2}, \vec{v}_{3}\right)$ : strongtype $(\mathrm{S})$, weak-type $(\mathrm{W})$ and expansion (U; unphysical). $\mathrm{W}$ are transonic right of + , supersonic left.

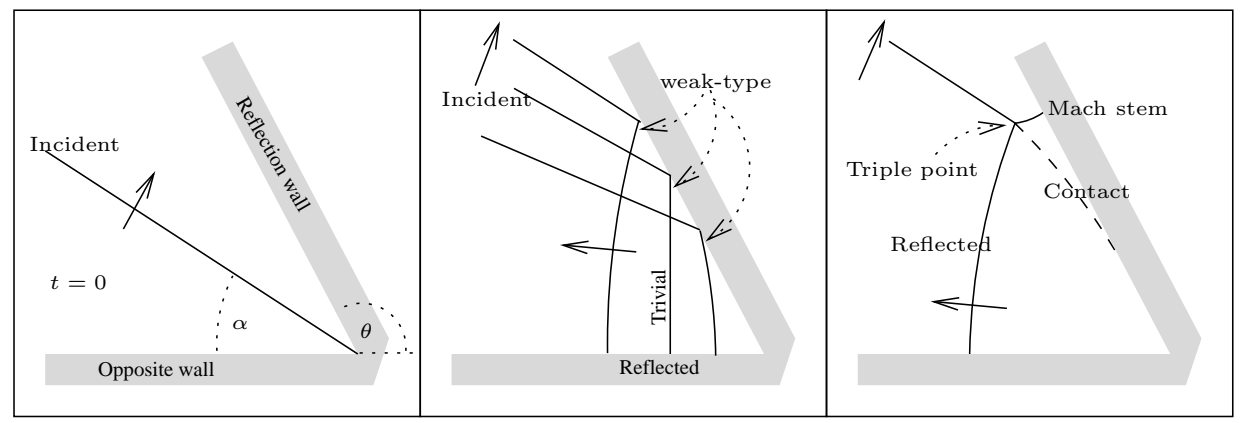

Figure 2: Left: initial data (from dotted area of Figure 3 second right). Center: $\mathrm{RR}$; we construct perturbations of the trivial case. Right: SMR

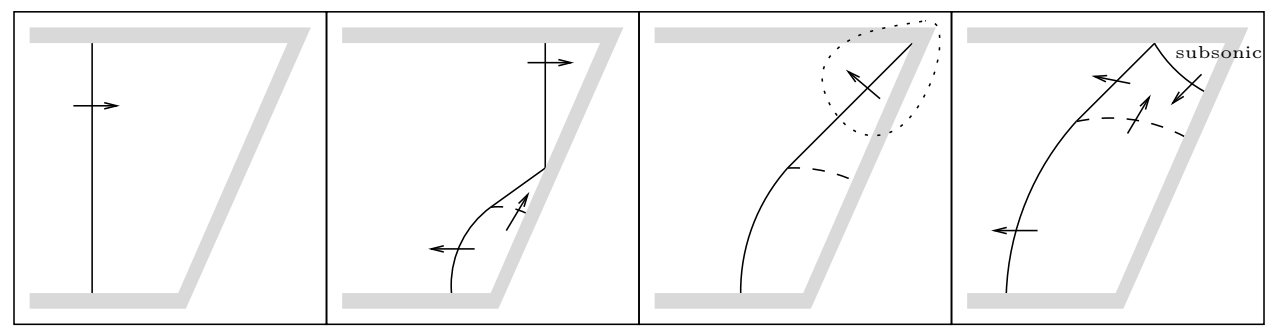

Figure 3: A shockwave breaks into a supersonic RR at the lower corner; the reflected shock breaks in the upper corner to produce our kind of subsonic RR for some time. 
In irregular reflections (IRR), incident and reflected shock are connected by a more or less complex interaction pattern which in turn connects to the solid surface by a third shock, called Mach stem. The most important irregular reflections are double, complex and single (see Figure 2 right) Mach reflection (MR); additional types have been discussed [13, 28, 16, 17, 26].

Some incident shocks allow more than one type of reflection. Assuming uniqueness for the problem at hand, only one of them can be extended to a global solution: a solution in the entire domain, satisfying all boundary and far-field conditions. A long-standing open question is to find the exact criterion that determines whether the solution is RR.

Among the criteria for appearance of RR that have been proposed (see [1, Section 1.5]), two are most important. The detachment criterion states that global RR appears generically whenever local RR is possible.

A physical argument motivates the second criterion: for a straight wall, all local $\mathrm{RR}$ and MR are trivially global solutions. But some of them could be unstable under perturbations, for example a bump in the 3-sector wall (Figure 1 left bottom). If so, then information is transmitted from the bump to the reflection point 2. For weak waves 3 that is possible if and only if the 3 -sector is subsonit 4 . Hence the sonic criterion: global RR appears generically if there is a supersonic local RR, but not otherwise. (Each criterion can also be formulated in other, slightly different ways.)

\subsection{Weak- and strong-type}

The velocity $\vec{v}_{2}$ in the 2 -sector in Figure 1 forms an angle $\tau$ with the wall; the reflected shock must turn this velocity by $\tau$ so that $\vec{v}_{3}$ is parallel to the wall, satisfying a slip boundary condition.

Keep the 2-sector data fixed while rotating the reflected shock in the reflection point. This yields a one-parameter family of velocities $\vec{v}_{3}$, forming a curve called shock polar (see Figure 1 right). For admissible shocks, $|\tau|$ cannot exceed $\tau_{*}$, the critical angle, which is a function of the Mach number $M_{2}$ and $\gamma$.

Throughout this paper we focus on polytropic equations of state so that the admissible part of the shock polar is strictly convex.

If the angle $\tau$ between wall and $\vec{v}_{2}$ is bigger than $\tau_{*}$, then local RR is theoretically impossible. If $\tau=\tau_{*}$, there is exactly one reflected shock, called critical-type.

\footnotetext{
${ }^{2}$ this is known as information condition or information argument

${ }^{3}$ but sufficiently strong shock waves can travel upstream against a supersonic flow

${ }^{4}$ the other sectors are always supersonic
} 
For $\tau<\tau_{*}$ however there are two, called weak-typ 5 and strong-type. We encounter another major issue in reflection: which of these two should occur? [10] have discussed this question for a different problem.

We call shocks transonic if the downstream side is subsonic, supersonic if both sides are supersonic. The weak shock is transonic for $\tau>\tau_{s}$ for some threshold $\tau_{s}<\tau_{*}$, supersonic otherwise; the strong-type shock is always transonic. In this paper we consider only transonic RR.

\subsection{Self-similar reflection problems}

Some variants of the reflection problem are self-similan 6 flow rather than steady.

In self-similar flow, density and velocity are functions of the similarity coordinates $(\xi, \eta)=(x / t, y / t)$ rather than $x, y$. Patterns grow linearly in time, with $t \downarrow 0$ corresponding to "zooming infinitely far away" whereas $t \uparrow+\infty$ is like "zooming into the origin" or "scaling up". Here inviscid models are easily justified because any flow feature eventually grows beyond the length scale where dissipate or kinetic phenomena matten7. Self-similar reflections occur naturally in many experiments (see Figure 3, 14, 2]).

We consider three parameters (see Figure 2 left): $M_{1}$, the 1-sector Mach are defined number, $\alpha$, clockwise angle from opposite wall to incident shock, and $180^{\circ}-\theta$, clockwise angle from opposite wall to reflection wall. The opposite wall passes 8 through $\vec{\xi}=\vec{v}_{2}$. Mach number and velocity are defined for an observer traveling in the reflection point.

For $t \downarrow 0$ this yields the initial data9 seen in Figure 2 left. Depending on $\theta$ either RR or MR appear.

If we choose the opposite wall perpendicular to the reflected shock, then local $\mathrm{RR}$ extends to a global trivial $R R$ (see Figure 2 center).

\footnotetext{
${ }^{5}$ The weak-type shock is relatively weaker than the strong-type shock, but their absolute strength can be arbitrarily small or large, so we prefer to use -type.

6 also called quasi-steady or pseudo-steady

${ }^{7}$ unless these small-scale phenomena trigger large-scale effects like turbulence, boundary layer separation etc.

8 to satisfy a slip condition on the opposite wall

${ }^{9}$ If the incident shock forms a right angle to the upstream wall, this problem is familiar 4. 8. Note that the nonvertical cases also arise from certain $t<0$ flows; in particular they can arise in simple experiments like Figure 3
} 

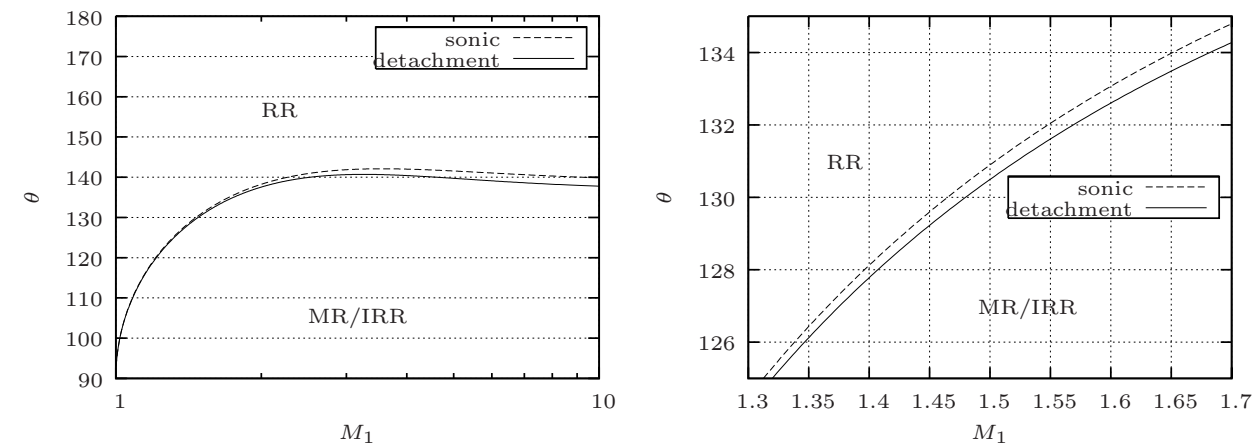

Figure 4: Left: $\theta_{d}, \theta_{s}$ for $\gamma=7 / 5$ potential flow and $\alpha=0$ in Figure 2 Right: detail.

\subsection{Transition}

The two transition criteria specify transition angles $\theta_{d}$ (detachment) and $\theta_{s}>\theta_{d}$ (sonic) depending on $L_{1}, \alpha, \gamma$. Global RR is predicted for larger $\theta$ and IRR for smaller $\theta$. Figure 4 compares the two criteria in the case of $\gamma=7 / 5$ polytropic potential flow.

To quote [1:

"For this reason it is almost impossible to distinguish experimentally between the sonic and detachment criteria."

Experimental and numerical accuracy are affected by viscosity/heat conduction 10, non-equilibrum effects, turbulence, surface roughness and other systematic errors as well as noise. The interaction of physical or numerical boundary layers with RR causes spurious Mach stems [29, Figure 7a] that make it look like $\mathrm{MR}$, however boundaries can be avoided by reflection into an interior problem.

Although the question has remained open, the sonic condition appears to have been favored by many researchers (including the author), at least for small $M_{1}$. As the recent survey [2] states,

"[...] the [criterion] which best agrees with pseudo-steady shock tube experimental data [...] suggests that in pseudo-steady flows $\mathrm{RR}$ terminates when the flow behind the reflection point, $\mathrm{R}[\ldots]$ becomes sonic in a frame of reference attached to R."

\footnotetext{
${ }^{10}$ Observations (e.g. 27 p. 142f) agree with our analytical solutions, so inviscid models are clearly suitable. Experiments [14 show that, although viscous/boundary layer effects can have a transient effect on the transition $\theta$, for sufficiently large times the transition is close to the inviscid predictions $\theta_{s}, \theta_{d}$.
} 
It should be noted that these quotes refer only to the classical case $\alpha=90^{\circ}$ and hence $\theta<90^{\circ}$, i.e. vertical incident shock (see Figure myreffig:experiment second left). Here we consider some cases with $\theta>90^{\circ}$ and $\alpha<90^{\circ}$ because they can be solved by linearization around trivial RR, i.e. small-data techniques. The classical case requires a large-data approach as in 10; this will be subject of future research. However, the nature of the question is the same in all cases: how is local RR affected by various kinds of perturbation. The classical perturbation occurs naturally in some experiments, but there is no other reason to favor it.

\subsection{Results}

We prove, for the self-similar reflection problem modeled with potential flow, that the sonic criterion is not universally correct. We use the following formulation 11:

Generic local transonic RR cannot extend into structurally stable global RR.

Instead, Theorem 2 shows:

1. Trivial weak-type transonic RR is structurally stable.

In particular, the parameter space has an open nonempty - hence generic subset with extendable local RR.

More importantly, we identify a physical reason for the failure of the sonic criterion. The information argument (see above) indeed goes a long way towards the correct answer. But interestingly, it is too restrictive in a subtle way:

2. For weak-type transonic reflections, downstream perturbations can reach the reflection point, but they decay to zero ${ }^{12}$ in the process.

This suggests that — although a proof is given only for particular parameters - the sonic criterion is incorrect for most, if not all, parameters, in particular including the classical case $\alpha=90^{\circ}$.

We demonstrate the principle for a particular variant of the reflection problem in potential flow. However, it will become clear during the course of the proof of Theorem 2 that gradient regularity near the reflection point is a local property of elliptic PDE and their boundary conditions in a domain corner. Hence the

\footnotetext{
${ }^{11}$ This version is a weak as possible, by considering "generic" instead of all, and by requiring structural stability.

${ }^{12}$ Here we mean decay in space, not in time.
} 
same principle applies to other steady or self-similar variants. Moreover, the same regularity effect occurs in isentropic and non-isentropic Euler flow.

A wider range of parameters, Euler flow and the validity of the detachment criterion will be discussed in separate articles. A third important criterion, the von Neumann criterion 13 , does not apply at all in potential flow 14 .

[10] previously provided a rigorous construction of supersonic weak-type reflections in a different problem. The techniques in this article are apparently sufficient to extend the construction to transonic cases.

Considerations analogous to the sonic criterion have also been used in studying the transitions between different types of Mach reflection (see the survey in [2] for details). Our findings suggest modifications to these proposals as well, by replacing subsonic-ness with weak-type in some way.

\subsection{Other remarks}

Many articles have considered dynamic stability 15 , trying to show that at the linear level weak-type reflections are stable while strong-type are not. However, numerical calculations [9, Figure 3] suggest that both types are dynamically stable.

15. has previously proposed a plausibility argument, based on pressure changes, for stability of weak-type transonic (and instability of strong-type) shocks. [5] show existence and structural stability of supersonic reflections from a wedge. 4. have constructed global supersonic $\mathrm{RR}$ for $\alpha=90^{\circ}$ and $\theta \approx 90^{\circ}$ as exact solutions of self-similar potential flow. 8] shows existence of global supersonic $\mathrm{RR}$ for a range of parameters that includes, in some cases, all $\theta>\theta_{s}$, proving that criteria more restrictive than sonic cannot be universally correct.

It has been proposed that both RR and MR may occur for the same parameters in steady flow, with hysteresis effects when parameters are changed (see e.g. [3]). In self-similar flow this would amount to non-uniqueness for an initial-value problem. Indeed, 6, 7] has found a set of initial data for the 2d Euler equations (both isentropic and non-isentropic) that appears to have two solutions, one theoretical, the other clearly different and observed in all numerical calculation:16 For isentropic Euler, a rigorous proof of a different non-uniqueness example has recently been proposed [19].

\footnotetext{
13 also referred to as mechanical equilibrum criterion in some contexts

${ }^{14}$ Even in Euler flow it applies only for large $M_{1}$, for example $M_{1}>2.2 \ldots$ for $\gamma=7 / 5$.

${ }^{15}$ stability under perturbations to the initial data

${ }^{16}$ In addition it is shown that the Godunov scheme can converge to either solution, depending on the grid.
} 
However, both results depend strongly on vorticity; uniqueness for the potential flow Cauchy problem is still expected and hysteresis is unlikely except as a transient phenomenon.

\section{Self-similar potential flow}

Here we prove technical results which are not previously available in the literature.

\section{$2.1 \quad$ Equations}

$2 \mathrm{~d}$ isentropic Euler flow is a PDE system for a density field $\rho$ and velocity field $\vec{v}$, consisting of the continuity equation

$$
\rho_{t}+\nabla \cdot(\rho \vec{v})=0
$$

and the momentum equations

$$
(\rho \vec{v})_{t}+\nabla \cdot(\rho \vec{v} \otimes \vec{v})+\nabla p=0
$$

The pressure $p$ is a strictly increasing smooth function of $\rho$. The sound speed $c$ is

$$
c=\sqrt{\frac{d p}{d \rho}(\rho)} .
$$

If we assume irrotationality

$$
\nabla \times \vec{v}
$$

then we may take

$$
\vec{v}=\nabla \phi
$$

for a scalar potential $\phi$. Assuming smooth flow, the momentum equations yield

$$
\rho=\pi^{-1}\left(A-\phi_{t}-\frac{1}{2}|\nabla \phi|^{2}\right)
$$

where $A$ is a global constant and where

$$
\frac{d \pi}{d \rho}=\frac{1}{\rho} \cdot \frac{d p}{d \rho}=\rho^{-1} c^{2} .
$$

The remaining continuity equation (1) is unsteady potential flow.

For any $t \neq 0$ we may change from standard coordinates $(t, x, y)$ to similarity coordinates $(t, \xi, \eta)$ with $\vec{\xi}=(\xi, \eta)=(x / t, y / t)$. A flow is self-similar if $\rho, \vec{v}$ are 
functions of $\xi, \eta$ alone, without explicit dependence on $t$. In potential flow that corresponds to the ansatz

$$
\phi(t, x, y)=t \psi(x / t, y / t) .
$$

By differentiating the divergence form (10) of potential flow and using (3) and (44), we obtain the form

$$
\left(c^{2} I-(\nabla \psi-\vec{\xi})^{2}\right): \nabla^{2} \psi=0 .
$$

Here $A: B$ is the Frobenius product $\operatorname{tr}\left(A^{T} B\right), \vec{w}^{2}:=\vec{w} \otimes \vec{w}=\vec{w} \vec{w}^{T}\left(\operatorname{not} \vec{w}^{T} \vec{w}\right)$ and $\nabla^{2}$ is accordingly the Hessian. In coordinates:

$$
\left(c^{2}-\left(\psi_{\xi}-\xi\right)^{2}\right) \psi_{\xi \xi}-2\left(\psi_{\xi}-\xi\right)\left(\psi_{\eta}-\eta\right) \psi_{\xi \eta}+\left(c^{2}-\left(\psi_{\eta}-\eta\right)^{2}\right) \psi_{\eta \eta}=0 .
$$

It is sometimes more convenient to use the pseudo-potential

$$
\chi:=\psi-\frac{1}{2}|\vec{\xi}|^{2}
$$

which yields

$$
\left(c^{2} I-\nabla \chi^{2}\right): \nabla^{2} \chi+2 c^{2}-|\nabla \chi|^{2}=0 .
$$

We choose $A=0$ so that

$$
\rho=\pi^{-1}\left(-\chi-\frac{1}{2}|\nabla \chi|^{2}\right) .
$$

(6) is manifestly translation-invariant. Translation is nontrivial: in $(t, x, y)$ coordinates it corresponds to a change of inertial frame

$$
\vec{v} \leftarrow \vec{v}-\vec{w}, \quad \vec{\xi}=\vec{x} / t \leftarrow \vec{\xi}-\vec{w},
$$

where $\vec{w}$ is the velocity of the new frame relative to the old one. Obviously the pseudo-velocity

$$
\vec{z}:=\nabla \chi=\nabla \psi-\vec{\xi}
$$

does not change.

Self-similar potential flow is mixed-type; the local type is determined by the coefficient matrix $c^{2} I-\nabla \chi^{2}$ which is positive definite if and only if $L<1$, where

$$
L:=\frac{|\vec{z}|}{c}=\frac{|\vec{v}-\vec{x} / t|}{c}
$$

is called pseudo-Mach number; for $L>1$ the equation is hyperbolic. 


\subsection{Shock conditions}

The weak solutions of potential flow are defined by the divergence-form continuity equation (11). Its self-similar form is

$$
\nabla \cdot(\rho \nabla \chi)+2 \rho=0 .
$$

The corresponding Rankine-Hugoniot condition is

$$
\rho_{u} z_{u}^{n}=\rho_{d} z_{d}^{n}
$$

where $u, d$ indicate the limits on the upstream and downstream side and $z^{n}, z^{t}$ are the normal and tangential component of $\vec{z}$. As the equation is second-order, we must additionally require continuity of the potential:

$$
\psi^{u}=\psi^{d}
$$

By taking a tangential derivative, we obtain

$$
z_{u}^{t}=z_{d}^{t}=: z^{t}
$$

Observing that $\sigma=\vec{\xi} \cdot \vec{n}$ is the shock speed, we obtain the more familiar form

$$
\begin{aligned}
\rho_{u} v_{u}^{n}-\rho_{d} v_{d}^{n} & =\sigma\left(\rho_{u}-\rho_{d}\right), \\
v_{u}^{t} & =v_{d}^{t}=: v^{t} .
\end{aligned}
$$

Fix the unit shock normal $\vec{n}$ so that $z_{u}^{n}>0$ which implies $z_{d}^{n}>0$ as well. To avoid expansion shocks we must require the admissibility condition $z_{u}^{n} \geq z_{d}^{n}$, which is equivalent to

$$
v_{u}^{n} \geq v_{d}^{n} .
$$

We chose the unit tangent $\vec{t}$ to be $90^{\circ}$ counterclockwise from $\vec{n}$.

By (12) the tangential components of the velocity are continuous across the shock, so the velocity jump is normal. Assuming $v_{u}^{n}>v_{d}^{n}$ (positive shock strength), we can express the shock normal as

$$
\vec{n}=\frac{\vec{v}_{u}-\vec{v}_{d}}{\left|\vec{v}_{u}-\vec{v}_{d}\right|} .
$$

\subsection{Shock polar}

In our problem the upstream regions are constant and determined. Let $\psi$ be the potential in the downstream region, $\psi^{I}$ the potential upstream (ditto for $\chi$, 
$\rho, \ldots)$. We substitute (14) into (8) to obtain the shock condition

$$
g(\nabla \psi, \psi, \vec{\xi}):=\left(\pi^{-1}\left(-\chi-\frac{1}{2}|\nabla \chi|^{2}\right) \nabla \chi-\rho^{I} \nabla \chi^{I}\right) \cdot \frac{\nabla \psi^{I}-\nabla \psi}{\left|\nabla \psi^{I}-\nabla \psi\right|}=0 .
$$

The shock polar (see Figure 1) is the curve of $\vec{v}_{d}$ that we obtain when holding the shock in a fixed $\vec{\xi}$ and keeping the upstream constant while varying the normal. For a fixed $\vec{\xi}, \nabla \chi^{I}$ is fixed and $\psi=\psi(\vec{\xi})=\psi^{I}(\vec{\xi})$ is fixed as well. Having eliminated the normal in (15), we see that the shock polar is the curve of solutions $\vec{v}=\nabla \psi$ of $g(\vec{v}, \psi, \vec{\xi})=0$. Hence the vector

$$
g_{\vec{v}}=\left(\frac{\partial g}{\partial v_{1}}, \frac{\partial g}{\partial v_{2}}\right)
$$

is normal to the shock polar, by the implicit function theorem. Omitting a positive scalar factor, it is given by the explicit formula

$$
g_{\vec{v}} \sim\left(1-\left(z_{d}^{n} / c\right)^{2}\right) \vec{n}-z^{t}\left(\frac{1}{z_{u}^{n}}+c^{-2} z_{d}^{n}\right) \vec{t},
$$

as we show in (23).

For transonic shocks, which are our focus, the downstream is elliptic, i.e. $1>$ $L_{d}=\left|\vec{z}_{d}\right| / c \geq z_{d}^{n} / c$. In this case the coefficient of $\vec{n}$ in (16) is necessarily positive, so $g_{\vec{v}} \neq 0$.

In Figure 1 right the leftmost point of the polar is a pseudo-normal shock: $z^{t}=0$. In this case $g_{\vec{v}}$ points in the same direction as $\vec{n}$, hence right. Therefore $g_{\vec{v}}$ is an inner norma17 to the admissible part of the shock polar.

In local RR the reflected shock must yield $\vec{v}_{3}$ parallel to the wall. In Figure 1 right, $\vec{v}_{d}$ for the weak shock (base in origin, tip in W) forms a blunt angle with inner normals of the shock polar whereas $\vec{v}_{d}$ for the strong shock (tip in $K$ ) forms a sharp angle. For the critical angle there is a single shock which is a limit of the weak and strong sides, so the angle is right (see $\tau_{*}$ in Figure 1 right). This motivates the following definition:

Definition 1. A shock is called weak-type (in a particular point $\vec{\xi}$ in self-similar coordinates) if

$$
g_{\vec{v}} \cdot \vec{z}_{d}<0
$$

strong-type if negative, critical-type if zero.

The definition has three pleasant properties: it coincides with the standard definition in the case of strictly convex polars, it generalizes the definition of weak/strong-type to non-convex cases 18 , and finally the sign condition is precisely what is needed for elliptic corner regularity.

\footnotetext{
${ }^{17}$ not necessarily unit

${ }^{18}$ In such cases, there may be three or more reflected shocks that yield $\vec{v}_{3}$ tangential to the wall.
} 


\subsection{Polytropic pressure}

Throughout the paper we consider only the standard polytropic pressure law:

$$
p(\rho)=\frac{c_{0}^{2} \rho_{0}}{\gamma}\left(\frac{\rho}{\rho_{0}}\right)^{\gamma}
$$

with $\gamma \in(1, \infty)$, where $c_{0}, \rho_{0}$ are constants. With this choice,

$$
c^{2}=c_{0}^{2}\left(\frac{\rho}{\rho_{0}}\right)^{\gamma-1} .
$$

Theorem 1. Consider arbitrary $c_{u}, \rho_{u}>0$ and $M_{u} \in(1, \infty)$ and set $\vec{v}_{u}=$ $\left(M_{u} c_{u}, 0\right)$. For each $\beta \in\left(-90^{\circ}, 90^{\circ}\right)$ there is a steady shock with downstream unit normal $\vec{n}=(\cos \beta, \sin \beta)$. Its downstream state $\rho_{d}, c_{d}, \vec{v}_{d}$ depends smoothly on $\beta$. Let $\tau$ be counterclockwise angle from $\vec{v}_{u}$ to $\vec{v}_{d}$. We restrict $|\beta|<\arccos \frac{1}{M_{u}}$ so that the shock is admissible.

Then the shock polar $\beta \mapsto \vec{v}_{d}$ is smooth and strictly convex, with $\partial_{\beta} \vec{v}_{d}$ nowhere zero.

There is an angle $\tau_{*} \in\left(0^{\circ}, 90^{\circ}\right)$ so that each $\tau \in\left(-\tau_{*}, \tau_{*}\right)$ is attained for two different $\beta$. The one with smaller $\left|\vec{v}_{d}\right|$ yields a strong-type shock, the other one weak-type. For $|\tau|=\tau_{*}$ they are identical and critical-type.

There is a $\tau_{s} \in\left(0, \tau_{*}\right)$ so that the weak-type shocks are supersonic for $|\tau|>\tau_{s}$, transonic for $|\tau|<\tau_{s}$. The other types are always transonic.

Proof. We refer to [10], especially Proposition 2.5.1, which establishes existence and smooth dependence of admissible shocks. By [10, (2.5.2)] $\partial_{\beta} \vec{v} \neq 0$ at all $\beta$.

As shown earlier, $g_{\vec{v}}$ in (16) is an inner normal to the shock polar everywhere. Multiply it with a positive factor to obtain $q=\vec{n}-A \vec{t}$ where

$$
A=\frac{v^{t}\left(1 / v_{u}^{n}+M_{d}^{n} / c_{d}\right)}{1-\left(M_{d}^{n}\right)^{2}} .
$$

$A$ is decreasing in $\beta \leq 0$, because by [10, Proposition 2.5.1] $c_{d}>0$ is increasing, $v_{u}^{n}>0$ is increasing, $M_{d}^{n}>0$ is decreasing, $v^{t}>0$ is decreasing. Hence $\partial_{\beta} A \leq 0$. Moreover

$$
\partial_{\beta} q=A \vec{n}+\left(1-\left(\partial_{\beta} A\right)\right) \vec{t},
$$

$(\vec{t}$ is counterclockwise from $\vec{n})$, so

$$
q \times \partial_{\beta} q=1-\partial_{\beta} A+A^{2}>0 .
$$

This implies that the upper half of the shock polar is strictly convex. By vertical symmetry and smoothness the entire polar is strictly convex. 
The shock polar is compact when adding the "vanishing" shock $\vec{v}_{d}=\vec{v}_{u}$. Moreover $|\tau|<90^{\circ}$, so there is a maximum $\tau_{*} \in\left(0^{\circ}, 90^{\circ}\right)$. By convexity there are exactly two points on the polar for $|\tau|<\tau_{*}$, which are the intersections of the line of multiples of $\vec{v}_{d}$ with the polar. As $g_{\vec{v}}$ is an inner normal, necessarily $g_{\vec{v}} \cdot \vec{v}_{d}>0$ for the point closer to the origin (strong-type), with opposite sign for the other (weak-type).

$$
g_{\vec{v}} \cdot \vec{v}=\left(1-\left(v_{d}^{n} / c_{d}\right)^{2}\right) v_{d}^{n}-\left(v^{t}\right)^{2}\left(1 / v_{u}^{n}+c_{d}^{-2} v_{d}^{n}\right)=v_{d}^{n}\left(\left(1-M_{d}^{2}\right)-\frac{\left(v^{t}\right)^{2}}{v_{d}^{n} v_{u}^{n}}\right) .
$$

If $M_{d} \geq 1$, then the right-hand side is negative, so the shock is weak-type. By Proposition 2.5.1, $M_{d}$ is strictly decreasing in $|\beta|$, so there is a unique $\tau_{s}$ so that the weak-type shock is transonic for $|\tau|>\tau_{s}$, supersonic for $|\tau|<\tau_{s}$.

\section{Perturbations of weak trivial RR}

\subsection{Coordinate transform}

We consider a trivial RR as in Figure 2 center or Figure 6 left. All lines and curves exclude endpoints by default. We use the following notation (see Figure 6 left): Let $\hat{B}$ be the reflection wall, $\hat{A}$ the opposite wall, $W$ the open convex cone enclosed by them. Let $\vec{n}_{A}, \vec{n}_{B}$ be the outer (with respect to $W$ ) unit normals of $\hat{A}, \hat{B}$. Let the origin the the corner between $A, B$. Let $S$ the reflected shock, $\vec{\xi}_{A}=\left(\xi_{A}, 0\right), \vec{\xi}_{B}=\left(\xi_{B}, \eta_{B}\right)$ (note $\left.\xi_{A}=\xi_{B}\right)$ the points where it meets $\hat{A}$ resp. $\hat{B}$. Let $A, B$ be the segments of $\hat{A}, \hat{B}$ from the corner $(0,0)$ to $\vec{\xi}_{A}, \vec{\xi}_{B}$; let $\Omega$ be the triangle enclosed by $A, B, S$.

The velocity in $\Omega$ is zero in the chosen coordinates, so the velocity potential $\psi$ is constant $=\psi^{0}$ in $\Omega$. Let $\vec{v}_{I}=\left(v_{I}^{x}, 0\right)$ be the 2-sector, $\psi^{I}$ the corresponding potential.

In self-similar flow, $\Omega$ is a uniformly elliptic region whereas the rest of $W$ is uniformly hyperbolic.

The shock is a free boundary. To linearize the problem, we first devise a transform from $\vec{\xi}=(\xi, \eta)$ to fixed coordinates $\vec{\sigma}=(\sigma, \zeta)$.

Given a function $\psi \in C^{2}(\Omega) \cap C^{1}(\bar{\Omega})$. Consider a ray starting in the origin and passing through $\left(\xi_{A}, \zeta\right) \in S$. $\psi^{I}$ is strictly monotone along any such ray, so there is a unique point $\vec{\xi}$ with

$$
\psi^{I}(\vec{\xi})=\psi\left(\xi_{A}, \zeta\right)
$$


$(\sigma, \zeta) \in \Omega$ is mapped to $\left(\frac{\xi \xi_{A}}{\sigma}, \frac{\eta \xi_{A}}{\sigma}\right)$.

This coordinate transform allows to state our problem in a fixed domain $\Omega$. By (18), $\psi$ mapped to $\vec{\xi}$ coordinates satisfies the first shock condition, $\psi=\psi^{I}$, automatically. Then (15) can be used as the second shock condition.

\subsection{Linearization}

We regard our problem as an operator equation

$$
F(\psi)=0
$$

where $F: X \rightarrow Y, X, Y$ Banach spaces with $X \subset C^{2}(\Omega) \cap C^{1}(\bar{\Omega})$. The map is the composition of three steps: first $\psi \in X$ is transformed from $(\sigma, \zeta)$ to $\vec{\xi}$ coordinates, then mapped to the tuple

$$
\begin{array}{lr}
\left(c^{2} I-\nabla \chi^{2}\right): \nabla^{2} \psi, & {[\text { Interior] }} \\
\nabla \psi_{\mid A} \cdot \vec{n}_{A}, & {[\text { Slip condition at } A]} \\
\nabla \psi_{\mid B} \cdot \vec{n}_{B}, & \text { [Slip condition at } B] \\
g(\nabla \psi, \psi, \vec{\xi})) . & \text { [Shock condition] }
\end{array}
$$

Finally, pull back this tuple to $(\sigma, \zeta)$ coordinates.

$X$ will be specified later since we have to consider an entire scale of such spaces. $F$ will be a nonlinear $C^{1}$ map from $X$ to $Y$. We intend to apply the implicit function theorem. To this end we need to study the Fréchet derivative $F^{\prime}\left(\psi^{0}\right)$ of $F$ with respect to $\psi$ at $\psi=\psi^{0}$.

The derivative is computed by considering a first variation $\psi^{\prime} \in X$ of $\psi^{0}$. We consider $\psi(\vec{\sigma})=\psi^{0}+t \psi^{\prime}(\vec{\sigma})$ and compute the derivative $\frac{d}{d t}$ of $F(\psi)$ and other expressions, evaluated at $t=0$. This derivative will be written $\vec{\xi}^{\prime}, \rho^{\prime}, F(\psi)^{\prime}$, etc. Obviously the usual calculus rules apply.

The following calculations are quite similar to [10, Proposition 4.14.3]. The results are simplified by two facts: $\psi=\psi^{0}$ yields an identity $(\xi, \eta)=(\sigma, \zeta)$, and $\nabla \psi^{0}=\nabla^{2} \psi^{0}=0$.

All derivatives are evaluated at $\psi=\psi^{0}$; we omit arguments where they are clear from the context. 


$$
\begin{aligned}
\left(\nabla_{\vec{\xi}} \psi\right)^{\prime} & =\left(\nabla_{\vec{\xi}} \vec{\sigma}^{T} \nabla_{\vec{\sigma}} \psi\right)^{\prime} \\
& =\left(\nabla_{\vec{\xi}} \vec{\sigma}^{T}\right)^{\prime} \underbrace{\nabla_{\vec{\sigma}} \psi}_{=0}+\nabla_{\vec{\xi}} \vec{\sigma}^{T}\left(\nabla_{\vec{\sigma}} \psi\right)^{\prime}=\nabla_{\vec{\xi}} \vec{\sigma}^{T} \nabla_{\vec{\sigma}} \psi^{\prime}=\nabla_{\vec{\xi}} \psi^{\prime} . \\
\left(\nabla_{\vec{\xi}}^{2} \psi\right)^{\prime}= & \left(\sum_{k} \frac{\partial \psi}{\partial \sigma^{k}} \nabla_{\vec{\xi}}^{2} \sigma^{k}+\nabla_{\vec{\xi}} \vec{\sigma}^{T} \nabla_{\vec{\sigma}}^{2} \psi \nabla_{\vec{\xi}}^{T} \vec{\sigma}\right)^{\prime} \\
= & \left(\sum_{k} \frac{\partial \psi}{\partial \sigma^{k}}\right)^{\prime} \nabla_{\vec{\xi}}^{2} \sigma^{k}+\sum_{k} \underbrace{\frac{\partial \psi}{\partial \sigma^{k}}}_{=0}\left(\nabla_{\vec{\xi}}^{2} \sigma^{k}\right)^{\prime} \\
& +\left(\nabla_{\vec{\xi}} \vec{\sigma}^{T}\right)^{\prime} \underbrace{\nabla_{\vec{\sigma}} \psi}_{=0} \nabla_{\vec{\xi}}^{T} \vec{\sigma}+\nabla_{\vec{\xi}} \vec{\sigma}^{T}\left(\nabla_{\vec{\sigma}}^{2} \psi\right)^{\prime} \nabla_{\vec{\xi}}^{T} \vec{\sigma}+\nabla_{\vec{\xi}} \vec{\sigma}^{T} \underbrace{\nabla_{\vec{\sigma}}^{2} \psi}_{=0}\left(\nabla_{\vec{\xi}}^{T} \vec{\sigma}\right)^{\prime} \\
= & \sum_{k} \frac{\partial \psi^{\prime}}{\partial \sigma^{k}} \nabla_{\vec{\xi}}^{2} \sigma^{k}+\nabla_{\vec{\xi}} \vec{\sigma}^{T} \nabla_{\vec{\sigma}}^{2} \psi^{\prime} \nabla_{\vec{\xi}}^{T} \vec{\sigma}=\nabla_{\vec{\xi}}^{2} \psi^{\prime}
\end{aligned}
$$

Fréchet derivative of the interior equation:

$$
\begin{aligned}
0 & =\left(c^{2} I-\left(\nabla_{\vec{\xi}} \chi\right)^{2}\right)^{\prime}: \underbrace{\nabla_{\vec{\xi}}^{2} \psi}_{=0}+\left(c^{2} I-\left(\nabla_{\vec{\xi}} \chi\right)^{2}\right):\left(\nabla_{\vec{\xi}}^{2} \psi\right)^{\prime} \\
& =\left(c^{2} I-\left(\nabla_{\vec{\xi}} \chi\right)^{2}\right): \nabla_{\vec{\xi}}^{2} \psi^{\prime}=\left(c^{2} I-\vec{\xi}^{2}\right): \nabla_{\vec{\xi}}^{2} \psi^{\prime}
\end{aligned}
$$

The resulting right-hand side is a linear elliptic operator without zeroth-order term, applied to $\psi^{\prime}$. The classical maximum principle shows that $\psi^{\prime}$ cannot have a minimum in the interior.

The wall conditions linearize to

$$
\nabla \psi^{\prime} \cdot \vec{n}=0 .
$$

For the shock condition, we consider (15). First, hold $\vec{\xi}, \psi$ fixed and very $\nabla_{\vec{\xi}} \psi$. The variation of the normal expression (14) is

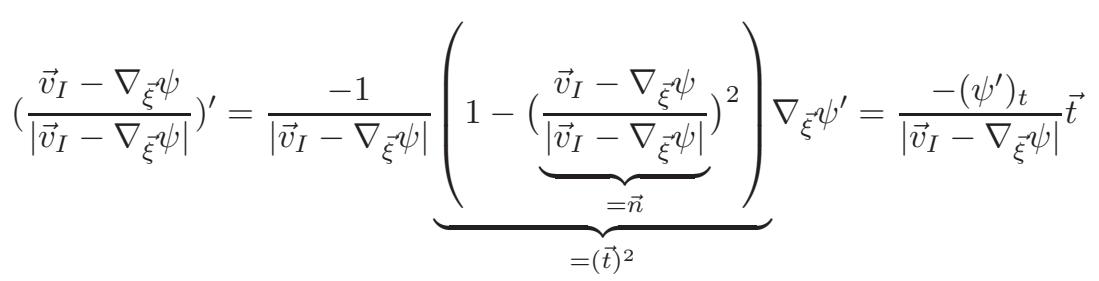


Moreover,

$$
\begin{aligned}
\left(\rho \nabla_{\vec{\xi}} \chi-\rho^{I} \nabla_{\vec{\xi}} \chi^{I}\right)^{\prime} & \stackrel{\text { 灵 }}{=}\left(\pi^{-1}\left(-\chi-\frac{1}{2}\left|\nabla_{\vec{\xi}} \chi\right|^{2}\right) \nabla_{\vec{\xi}} \chi\right)^{\prime} \\
& \stackrel{\text { 崮 }}{=} \rho\left(I-c^{-2} \nabla_{\vec{\xi}} \chi^{2}\right) \nabla \psi^{\prime} .
\end{aligned}
$$

Both combined, we use the shock relations $\rho_{I}=\rho \chi_{n} / \chi_{n}^{I}$ and $\chi_{t}=\chi_{t}^{I}$ to compute

$$
\begin{aligned}
\left(g\left(\nabla_{\vec{\xi}} \psi, \psi, \vec{\xi}\right)\right)^{\prime} & =\left(\left(\rho \nabla_{\vec{\xi}} \chi-\rho^{I} \nabla_{\vec{\xi}} \chi^{I}\right) \cdot \frac{\vec{v}_{I}-\nabla_{\vec{\xi}} \psi}{\left|\vec{v}_{I}-\nabla_{\vec{\xi}} \psi\right|}\right)^{\prime} \\
& =\left(\left(\rho \nabla_{\vec{\xi}} \chi-\rho^{I} \nabla_{\vec{\xi}} \chi^{I}\right)\right)^{\prime} \cdot \vec{n}+\left(\rho \nabla_{\vec{\xi}} \chi-\rho^{I} \nabla_{\vec{\xi}} \chi^{I}\right) \cdot\left(\frac{\vec{v}_{I}-\nabla_{\vec{\xi}} \psi}{\left|\vec{v}_{I}-\nabla_{\vec{\xi}} \psi\right|}\right)^{\prime} \\
& =\rho \vec{n}^{T}\left(I-c^{-2} \nabla_{\vec{\xi}} \chi^{2}\right) \nabla_{\vec{\xi}} \psi^{\prime}-\frac{\rho \chi_{t}-\rho^{I} \chi_{t}^{I}}{\left|\vec{v}_{I}-\nabla_{\vec{\xi}} \psi\right|} \psi_{t}^{\prime} \\
& =\underbrace{\rho\left(\left(1-c^{-2} \chi_{n}^{2}\right) \vec{n}-\chi_{t}\left(\frac{1}{\chi_{n}^{I}}+c^{-2} \chi_{n}\right) \vec{t}\right)}_{=: g_{\vec{v}}} \cdot \nabla_{\vec{\xi}} \psi^{\prime}
\end{aligned}
$$

Now we hold $\nabla_{\vec{\xi}} \psi$ fixed and vary $\psi \cdot \psi=\psi^{I}=\psi^{I}(0,0)+v_{I}^{x} \xi$ on the shock, so we can use

$$
\xi^{\prime}=\left(v_{I}^{x}\right)^{-1} \psi^{\prime} .
$$

Moreover, the variation of the "normal" $\vec{v}_{I}-\nabla_{\xi} \psi$ is zero here, so:

$$
\begin{aligned}
(g)^{\prime} & =\left(\pi^{-1}\left(-\psi+\frac{1}{2}|\vec{\xi}|^{2}-\frac{1}{2}\left|\nabla_{\vec{\xi}} \psi-\vec{\xi}\right|^{2}\right)\left(\nabla_{\vec{\xi}} \psi-\vec{\xi}\right)-\rho^{I}\left(\vec{v}_{I}-\vec{\xi}\right)\right)^{\prime} \cdot \underbrace{\vec{n}}_{=(1,0)} \\
& =\left(\rho c^{-2} \cdot(-\psi^{\prime}+\underbrace{\vec{\xi} \cdot\left(\vec{\xi}^{\prime}\right)+\left(\nabla_{\vec{\xi}} \psi-\vec{\xi}\right) \cdot(\vec{\xi})^{\prime}}_{=\nabla_{\vec{\xi}} \psi \cdot(\vec{\xi})^{\prime}=0}) \nabla_{\vec{\xi}} \chi-\rho(\vec{\xi})^{\prime}+\rho_{I}(\vec{\xi})^{\prime}\right) \cdot \underbrace{\vec{n}}_{=(1,0)} \\
& =-\rho c^{-2} \chi_{n} \psi^{\prime}+\left(\rho_{I}-\rho\right) \xi^{\prime} \stackrel{24}{=}_{=}^{2}-\rho c^{-2} \chi_{n} \psi^{\prime}+\frac{\rho_{I}-\rho}{v_{I}^{x}} \psi^{\prime} \\
& =-\rho\left(\frac{1}{\chi_{n}^{I}}+c^{-2} \chi_{n}\right) \psi^{\prime}
\end{aligned}
$$

It turns out that $\eta^{\prime}$ does not appear in the final form, so the details of the coordinate transform do not matter at all!

Altogether, when varying $\nabla \psi$ and $\psi$ at the same time, the shock relations linearize to

$$
(g)^{\prime}=g_{\vec{v}} \cdot \nabla_{\vec{\xi}} \psi^{\prime}-\rho\left(\frac{1}{\chi_{n}^{I}}+c^{-2} \chi_{n}\right) \psi^{\prime}
$$




\subsection{Kernel}

Proposition 2. For any $X \subset C^{2}(\Omega) \cap C^{1}(\bar{\Omega})$,

$$
\operatorname{dim} \operatorname{ker} F^{\prime}\left(\psi^{0}\right) \leq 1 .
$$

If $=1$, then it is spanned by a function $\psi^{\prime}$ that satisfies

$$
\psi^{\prime}\left(\vec{\xi}_{B}\right) \neq 0 .
$$

Proof. Assume the kernel is nontrivial. Let $\psi^{\prime}$ be a nonzero element.

Consider a positive local maximum (with respect to $\bar{\Omega}$ ) of $\psi^{\prime}$ at $S \cup\left\{\vec{\xi}_{A}\right\}$. A maximum at $S$ requires $\psi_{t}^{\prime}=0$; for a maximum in $\vec{\xi}_{A}$ this is already implied by the boundary condition $\psi_{n}^{\prime}=0$ on $A$, by $C^{1}$ regularity in the corner, since $A$ and $S$ meet at a right angle. The coefficients of $-\psi_{n}^{\prime}$ and $\psi^{\prime}$ in (25), the linearization of the shock condition, have opposite sign. Therefore $\psi^{\prime}>0$ in the maximum point implies $-\psi_{n}>0$ which is incompatible with a local maximum $(\vec{n}$, the downstream normal, is an inner normal for $\Omega$ ). By the same argument a local negative minimum is ruled out.

This implies in particular that $\psi^{\prime}$ cannot be constant.

$\psi^{\prime}$ satisfies (20), the linearization of the interior PDE, so by the classical strong maximum principle $\psi^{\prime}$ cannot have a local extremum in $\Omega$ unless it is constant. By the Hopf lemma, the wall boundary condition (21) does not allow a local extremum at $A$ or $B$ unless $\psi^{\prime}$ is constant.

Assume $\psi^{\prime}$ has a global maximum in 0 (wall-wall corner). Let $B_{\epsilon}(0)$ be the ball with radius $\epsilon$ centered in 0 and abbreviate $U:=B_{\epsilon}(0) \cap \Omega, I:=\partial B_{\epsilon}(0) \cap \Omega$. For sufficiently small $\epsilon>0, \bar{I} \subset \Omega \cup A \cup B$, so as shown above $\psi^{\prime}$ cannot attain a maximum on $\bar{I}$. Therefore $\psi^{\prime}(0)>\max _{\bar{I}} \psi^{\prime}$.

$\hat{\psi}:=\psi^{\prime}-\psi^{\prime}(0)+\delta \xi$ is a supersolution for $\delta \geq 0$ :

$$
\left(I-c^{-2} \vec{\xi}^{2}\right): \nabla^{2} \hat{\psi}=\left(I-c^{-2} \vec{\xi}^{2}\right): \nabla^{2} \psi^{\prime}=0
$$

by linearity, $\hat{\psi}_{n}=0$ on $A$ and $\hat{\psi}_{n}=(\delta \xi)_{n}>0$ or 19 . Therefore $\hat{\psi}$ does not attain extrema in $U$. For $\delta>0$ sufficiently small,

$$
\max _{\bar{I}} \hat{\psi}=\max _{\bar{I}} \psi^{\prime}-\psi^{\prime}(0)+\delta \xi>0,
$$

while $\hat{\psi}(0)-\psi^{\prime}(0)=0$, so the minimum of $\hat{\psi}$ over $\bar{U}$ is attained in 0 . Therefore $\hat{\psi}_{\xi}(0) \leq 0$, hence $\psi_{\xi}^{\prime}(0) \leq-\delta<0$. But the boundary conditions $\psi_{n}^{\prime}=0$ on

\footnotetext{
${ }^{19}$ note $\theta>90^{\circ}$ for trivial $\mathrm{RR}$
} 
$\bar{A}, \bar{B}$ combine to $\nabla \psi^{\prime}(0)=0$ - contradiction. Hence $\psi^{\prime}$ cannot have a global maximum in 0 ; minima are ruled out analogously.

Since $\psi^{\prime}$ is nonzero, it must have a positive maximum or negative minimum somewhere. As we have shown that is not possible except in $\vec{\xi}_{R}$.

For any two elements of the kernel, a suitable linear combination is zero in $\vec{\xi}_{R}$, hence zero everywhere. Thus the kernel cannot have dimension higher than 1.

\subsection{Type and Fredholm index}

Proposition 3. Consider the eigenvalues of the operator pencil for $F^{\prime}\left(\psi^{0}\right)$ in the reflection corner $\vec{\xi}_{B}$ (see Section 4.2). There is an eigenvalue $\lambda_{0}=\alpha_{0}+i \beta_{0}$ of multiplicity 1 with least nonnegative $\beta_{0}$, and

$$
\beta_{0} \begin{cases}\in(0,1), & \text { if the shock is strong-type in } \vec{\xi}_{B}, \\ =1, & \text { for critical-type, } \\ >1, & \text { for weak-type. }\end{cases}
$$

Proof. The operator $F^{\prime}\left(\psi_{0}\right)$, with coefficients frozen in $\vec{\xi}_{B}$, consists of the interior operator $\left(I-c^{-2} \vec{\xi}_{B}^{2}\right): \nabla^{2} \psi^{\prime}$ and the boundary operators $\nabla \psi^{\prime} \cdot \vec{n}_{B}$ and $g_{\vec{v}} \cdot \nabla \psi^{\prime}$. We choose a linear coordinate transform so that the interior operator is mapped into $\Delta \psi^{\prime}$. This transform is a dilation in the $B$ direction.

Consider polar coordinates $(r, \phi)$ centered in $\vec{\xi}_{B}$. Let $\Gamma_{2}=B, \Gamma_{1}=S$, then the boundary operators take the form (31) with $\gamma_{2}=90^{\circ}$ (Neumann) and (see Figure 5)

$$
\gamma_{1} \begin{cases}\in\left(90^{\circ}, \phi_{2}-\phi_{1}+90^{\circ}\right), & \text { for strong-type, } \\ =\phi_{2}-\phi_{1}+90^{\circ}, & \text { for critical-type, } \\ \in\left(\phi_{2}-\phi_{1}+90^{\circ}, 180^{\circ}\right), & \text { for weak-type. }\end{cases}
$$

To see this, note that $\nabla \chi^{0}=\nabla \psi^{0}-\vec{\xi}=-\vec{\xi} \| B$ on $B$. For a weak-type shock (Definition (1), $g_{\vec{v}} \cdot \nabla \chi^{0}<0$, so $\vec{n}_{B} \times g_{\vec{v}}>0$. This property is preserved under dilation along $B$, so $\gamma_{1}>\phi_{2}-\phi_{1}+90^{\circ}$ (see Figure 5 left). The other types are analogous.

Now (32) immediately implies (26).

Proposition 4. Consider the eigenvalues $\lambda=\alpha+i \beta$ of the operator pencil of $F^{\prime}\left(\psi^{0}\right)$ in the $A, B$ and $A, S$ corner. The eigenvalue with least nonnegative $\beta$ is 


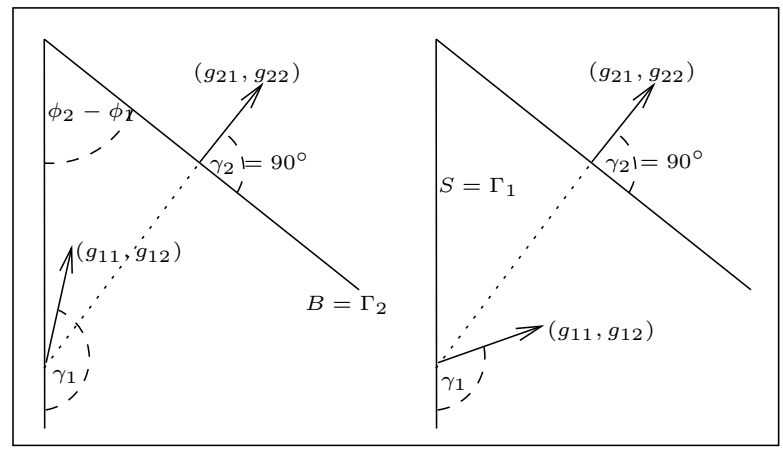

Figure 5: Corner limits of the top-order parts $\left(g_{k 1}, g_{k 2}\right) \cdot \nabla \psi^{\prime}$ of the boundary operators. Left: weak-type shock. Right: strong-type shock: solutions need not be $C^{1}$ in the corner. A critical-type shock has $\left(g_{11}, g_{12}\right)$ exactly perpendicular to $B$.

$\lambda_{0}=0$. The eigenvalue with next lowest nonnegative $\beta$ is $\beta_{1}=1 /\left(1-\theta / 180^{\circ}\right)>$ 1 in the $A, B$ corner and $\beta_{1}=2$ in the $A, S$ corner; their multiplicity is 1 .

Proof. In the $A, B$ corner the interior operator is $\Delta$, with Neumann boundary operators $\partial_{n}$ (so $\gamma_{1}=\gamma_{2}=90^{\circ}$ in the notation of Section 4.2), so the calculation is straightforward. Take $\Gamma_{1}:=B, \Gamma_{2}:=A$. $\phi_{1}=\theta, \phi_{2}=\pi$, then by 32

$$
\beta_{0}=0, \quad \beta_{1}=\frac{\pi}{\pi-\theta} .
$$

In the $A, S$ corner the slip condition on $A$ yields $\chi_{\eta}=0$, so the interior operator is $\left(1-\chi_{\xi}^{2}\right) \partial_{\xi \xi}+\partial_{\eta \eta}$ which becomes $\Delta$ by dilation. Moreover by (25) both boundary operators are $\partial_{n}$, which are not changed by dilation. Hence $\beta_{1}=2$ by (32).

Proposition 5. Consider a weak-type trivial reflection. Let $s \in(2,3)$. For $\epsilon>0$ sufficiently small, the Fredholm index of $F^{\prime}\left(\psi^{0}\right)$ as a map from $X_{1+\epsilon}^{s}$ to $Y_{1+\epsilon}^{s}$ (defined in Section 4.2) is 1.

Proof. Consider the operator $\Delta-I$ on the triangle $\Omega_{0}$ with Neumann boundary operators $\partial_{n}$ on $A, B, S$. [20, Theorem 1.4] yields $s \in(2,3)$ and $\epsilon \in(0, s-2)$ so that the operator is a linear isomorphism on $X_{1+3 \epsilon}^{s}$ onto $Y_{1+3 \epsilon}^{s}$. The space $X_{1+3 \epsilon}^{s}$ defined in the present paper corresponds to $H_{s}^{(-1-3 \epsilon)}$ in his notation, except that his weights are with respect to $\partial \Omega$, not $\Sigma$. But $\partial \Omega-\Sigma$ consists of line segments, so classical potential theory20 [11, Lemma 6.27] extends his result to our case.

\footnotetext{
${ }^{20}$ moreover $s$ determines only regularity away from the corners, so it can be improved to any $s>2$
} 


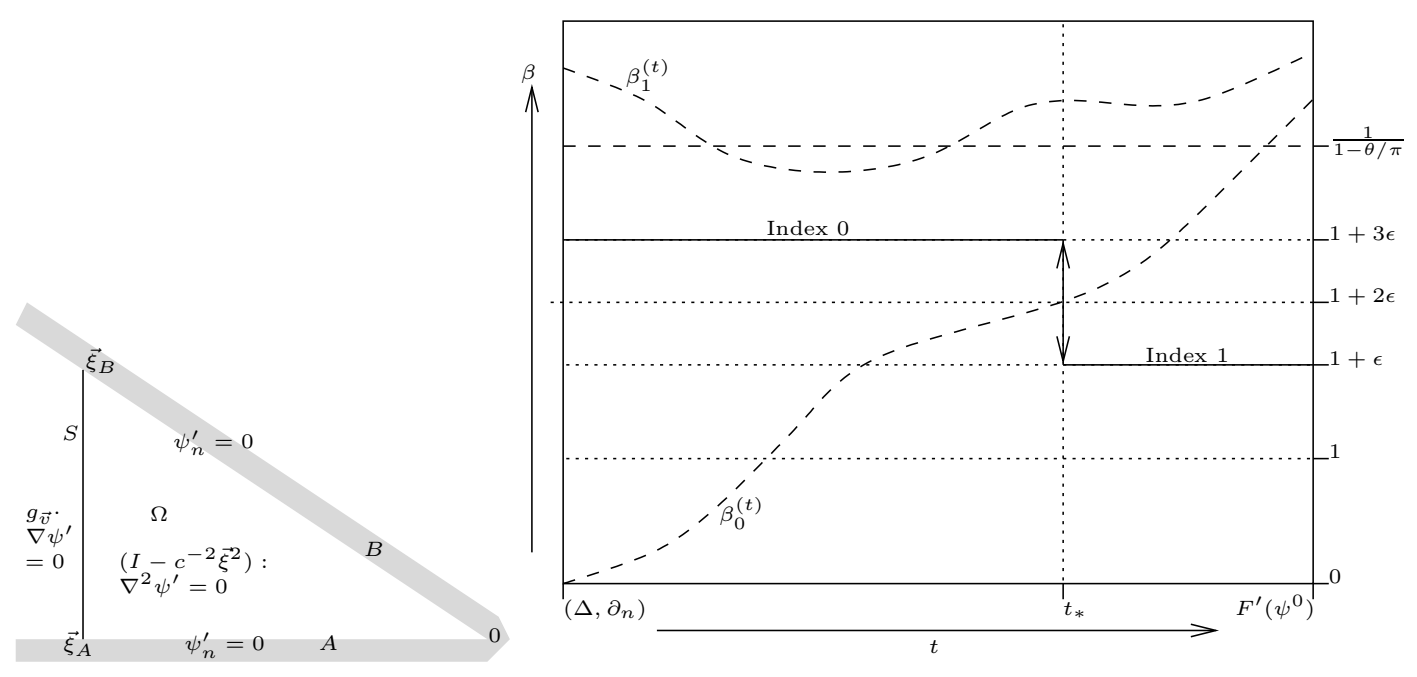

Figure 6: Left: notation and linearized operator. Right: each point represents an operator $T^{(t)}: X_{\beta}^{s} \rightarrow Y_{\beta}^{s}$ ( $t$ horizontal axis, $\beta$ vertical axis). The operators are Fredholm except on the dashed curves, where some corner operator pencils has an eigenvalue. Between the curve the index is constant. Across the curves the index jumps by the eigenvalue multiplicity.

Now we choose a family of operators $t \mapsto T^{(t)}$ so that $T^{(0)}=\left(\Delta-I, \partial_{n}, \partial_{n}, \partial_{n}\right)$ and $T^{(1)}=F^{\prime}\left(\psi^{0}\right)$ (see Figure 6). The family is chosen continuous in $t$ with respect to all operator norms we consider, which is easily achieved by choosing a continuous family of coefficients for interior and boundary operators. We choose the family so that $\beta_{0}, \beta_{1}$ in the $A, B$ and $A, S$ corners are constant in $t$ (Proposition 4). If $\beta_{j}^{(t)}(j=0,1)$ are the two lowest nonnegative imaginary parts of eigenvalues of the operator pencils in the $\vec{\xi}_{B}$ corner, then $t \mapsto \beta_{j}^{(t)}$ are continuous as well. $\beta_{0}^{(0)}=0$ whereas Proposition 3 shows that $\beta_{0}^{(1)}>1$. By choosing suitable coefficient families in the reflection corner we can make $\beta_{0}^{(t)}$ strictly increasing in $t$. Moreover $\beta_{1}^{(0)}, \beta_{1}^{(1)}>1$, so we can achieve $\beta_{1}^{(t)}>1$.

23. Theorem 6.3] yield 21 that $T^{(t)}: X_{1+\epsilon}^{s} \rightarrow Y_{1+\epsilon}^{s}$ is a Fredholm operator if $1+\epsilon$ is not the imaginary part of an operator pencil eigenvalue in any corner.

Choose $\epsilon \in(0,1)$ sufficiently small (not larger than above) so that $1+3 \epsilon<$ $\beta_{1}^{(t)}, 1 /\left(1-\theta / 180^{\circ}\right)$ for all $t$ (see Figure 6 right). Let $t_{*} \in(0,1)$ be such that $\beta_{0}^{\left(t_{*}\right)}=1+2 \epsilon$. Then $t \in\left[0, t_{*}\right] \mapsto T^{(t)}: X_{1+3 \epsilon}^{s} \rightarrow Y_{1+3 \epsilon}^{s}$ and $t \in\left[t_{*}, 1\right] \mapsto$ $T^{(t)}: X_{1+\epsilon}^{s} \rightarrow Y_{1+\epsilon}^{s}$ are both continuous families of Fredholm operators. By

\footnotetext{
21 Their weighted Hölder spaces are homogeneous; for our inhomogeneous spaces, $\oplus \Pi_{1}$ (set of polynomials of degree $\leq 1$ ) is added for each corner, which is only a finite-dimensional change.
} 
Fredholm theory the index of each family is constant. $T^{(0)}: X_{1+\epsilon}^{s} \rightarrow Y_{1+\epsilon}^{s}$ is an isomorphism, as shown above, i.e. has index 0 .

The interval $[1+\epsilon, 1+3 \epsilon]$ contains only one eigenvalue of a corner operator pencil of $T^{t_{*}}$, namely $\beta_{1}^{\left(t_{*}\right)}=1+2 \epsilon$; its multiplicity is 1 . Hence [23, Theorem 6.4] shows that $\operatorname{dim}\left(Z / X_{+}\right)=1$ in Proposition 8 , where we choose $X_{-}=X_{1+3 \epsilon}^{s}, Y_{-}=$ $Y_{1+3 \epsilon}^{s}, X_{+}=X_{1+\epsilon}^{s}, Y_{+}=Y_{1+\epsilon}^{s}$ and $A_{ \pm}=T^{\left(t_{*}\right)}$. Therefore ind $A_{-}-$ind $A_{+}=1$, so $T^{(1)}: X_{1+\epsilon}^{s} \rightarrow Y_{1+\epsilon}^{s}$ has index 1 .

Remark 6. The proof requires $\beta_{0}>1$, which is not satisfied for critical- or strong-type shocks in the reflection corner. This is the crucial difference to weak-type shocks. Note that the value of $\beta_{0}$ is a purely local property; the chosen far-field perturbation is not significant.

\subsection{Perturbation}

Theorem 2. Consider a weak-type trivial transonic $R R$, with parameters $\vec{p}_{0}=$ $\left(M_{1}, \theta, \alpha\right)$. There is a ball $U$ of radius $r>0$ around $\vec{p}_{0}$ so that there is another global weak-type $R R$ for any $\vec{p} \in U$.

Proof. Since the downstream state of a shock depends smoothly on the shock normal, location and upstream state, the shock polar varies smoothly with $M_{u}$. Necessarily $M_{2}>1$, so the incident shock is weak-type like the reflected shock. Therefore, sufficiently small perturbations $\vec{p}$ yield a new local $\mathrm{RR}$ which is close to the old one. In particular the perturbation of the reflection point is small and the reflected shock is still weak-type.

By Proposition 5 , the Fredholm index of $F^{\prime}\left(\psi^{0}\right)$ is 1 . By Proposition 2 the kernel has dimension 1 , so the codimension of the range is 0 . Therefore we can apply the implicit function theorem, with a single real free parameter. By Proposition 2 we can use $\psi\left(\vec{\xi}_{B}\right)$ as free parameter, which corresponds to changing the reflection point.

Therefore we obtain a new elliptic region for sufficiently small perturbations of the reflection point, while satisfying both shock conditions. After extending the solution to the entire domain by adding incident shock and hyperbolic regions, we have obtained a global transonic weak-type RR. 


\section{Corner domains}

Here we adapt some literature results to our case. For details, see [12, 23], 22 , and [24].

\subsection{Weighted Hölder spaces}

Consider a bounded open simply connected Lipschitz domain $\Omega \subset \mathbb{R}^{2}$. Let $\Gamma_{k}(k=1, \ldots, m)$ be pairwise disjoint line segments with (excluded) endpoints $y_{k-1}, y_{k}\left(\right.$ set $y_{0}:=y_{m}, \Gamma_{0}:=\Gamma_{m}$ for simplicity). Set $\Sigma:=\left\{y_{1}, \ldots, y_{m}\right\}$. Assume $\partial \Omega=\bigcup_{k=1}^{m} \bar{\Gamma}_{k}$. Let $\Gamma_{1}, \ldots, \Gamma_{m}$ pass around $\Omega$ clockwise, so that $\Omega$ lies counterclockwise from $\Gamma_{k}$ to $\Gamma_{k+1}$ near each corner $y_{k}$.

Definition 7. Let $\beta \in \mathbb{R}, s \in(0, \infty)-\mathbb{Z}$. Abbreviate $\bar{\Omega}_{r}:=\bar{\Omega}-B_{r}(\Sigma)$ where $B_{r}$ is the $r$-neighbourhood. For $u \in C^{s}(\bar{\Omega}, \Sigma)$ we define weighted Hölder norms

$$
\|u\|_{C_{\beta}^{s}(\bar{\Omega}, \Sigma)}:=\limsup _{r \downarrow 0} r^{s-\beta}\|u\|_{C^{s}\left(\bar{\Omega}_{r}\right)} .
$$

Then $C_{\beta}^{s}(\bar{\Omega}, \Sigma)$ is the set of $u$ with finite norm. The definitions for $\Gamma_{j}$ in place of $\Omega$ are analogous.

Non-integer $\beta$ corresponds to the lowest exponent of $r^{\beta}$ behaviour allowed in a corner; note that $C_{\beta}^{s}(\bar{\Omega}, \Sigma) \subset C^{\beta}(\bar{\Omega})$.

\subsection{Operator pencils}

Consider the operator of a linear second-order elliptic boundary value problem:

$$
\begin{aligned}
L(x) u & :=\sum_{i, j=1}^{2} a_{i j}(x) \frac{\partial^{2}}{\partial x_{i} \partial x_{j}} u+\sum_{i=1}^{2} b_{i}(x) \frac{\partial}{\partial x_{i}} u+c(x) u \quad \text { in } \Omega, \\
B_{k}(x) u & :=\sum_{i=1}^{2} g_{k i}(x) \frac{\partial}{\partial x_{i}} u+h_{k}(x) u \quad \text { on } \Gamma_{k}, k=1, \ldots, m .
\end{aligned}
$$

We assume that the coefficients $a_{i j}, b_{i}, c$ are smooth on $\bar{\Omega}$ and $g_{k i}, h_{k}$ smooth on $\bar{\Gamma}_{k}$. We write $B=\left(B_{1}, \ldots, B_{m}\right)$. Let $s \in(2, \infty)-\mathbb{Z}$; we use the convenient abbreviations

$$
X_{\beta}^{s}:=C_{\beta}^{s}(\bar{\Omega}, \Sigma), \quad Y_{\beta}^{s}:=C_{\beta-2}^{s-2}(\bar{\Omega}, \Sigma) \times \prod_{k=1}^{m} C_{\beta-1}^{s-1}\left(\bar{\Gamma}_{i}, \Sigma\right) .
$$


$(L, B): X_{\beta}^{s} \rightarrow Y_{\beta}^{s}$ is a continuous linear operator.

Whenever $L\left(y_{1}\right)$ is elliptic, we can find a linear invertible coordinate transformation so that the leading-order part of $L\left(y_{1}\right)$ transforms to the Laplace operator $\Delta$. In this new frame we consider polar coordinates $(r, \phi)$ centered in $y_{1}$. Let $\phi_{1}, \phi_{2}$ correspond to $\Gamma_{1}, \Gamma_{2}$; we normalize $\phi_{1} \in\left[0,360^{\circ}\right)$ and $\phi_{2} \in\left[\phi_{1}, \phi_{1}+360^{\circ}\right)$. The coordinate transformation from $(x, y)$ to $(t, \phi)$ with $t=\log r$ is conformal, hence preserves the Laplace operator, mapping the cone $\{(r, \phi): r>0, \phi \in$ $\left.\left(\phi_{1}, \phi_{2}\right)\right\}$ to an infinite strip $\mathbb{R} \times\left(\phi_{1}, \phi_{2}\right)$. The leading-order parts of $B_{k}\left(y_{1}\right)$ are

$$
\frac{\partial u}{\partial t} \cos \gamma_{k}+\frac{\partial u}{\partial \phi} \sin \gamma_{k}
$$

Here $\gamma_{k}$ is the counterclockwise angle from $\Gamma_{k}$ to the coefficient vector $\left(g_{k 1}, g_{k 2}\right)$ on the corresponding boundary (see Figure 5). We normalize $\gamma_{1} \in\left[0,180^{\circ}\right.$ ) and $\gamma_{2} \in\left(\gamma_{1}-180^{\circ}, \gamma_{1}\right]$.

Apply the Fourier-Mellin transform in $t$ to the homogeneous corner equation

$$
-\Delta_{(t, \phi)} u=\left(-i \partial_{t}\right)^{2}+\left(-i \partial_{\phi}\right)^{2}=0
$$

to obtain the operator pencil equation

$$
\left(-i \partial_{\phi}\right)^{2} \tilde{u}+\lambda^{2} \tilde{u}=0
$$

where $\lambda=\alpha+i \beta$ are the eigenvalues. The eigenfunctions yield well-known harmonic functions

$$
u(t, \phi)=\exp (\beta t) \sin (\beta \phi-\delta)=r^{\beta} \sin (\beta \phi-\delta) .
$$

Imposing homogeneous boundary conditions restricts this family to

$$
u(r, \phi)=r^{\beta_{\ell}} \sin \left(\beta_{\ell}\left(\phi-\phi_{1}\right)-\gamma_{1}\right)
$$

with

$$
\beta_{0}=-\frac{\gamma_{2}-\gamma_{1}}{\phi_{2}-\phi_{1}}, \quad \beta_{\ell}=\beta_{0}+\frac{\pi}{\phi_{2}-\phi_{1}} \ell \quad(\ell \in \mathbb{Z}) .
$$

The multiplicity of each eigenfunction is 1 , except when $\beta=0$ where it is 2 (for example in the case of two Neumann conditions there is another eigenfunction $u=t=\log r)$.

\subsection{Fredholm index jump}

Proposition 8. Consider Banach spaces $X_{+} \subset X_{-}$and $Y_{+} \subset Y_{-}$and Fredholm operators $A_{ \pm}: X_{ \pm} \rightarrow Y_{ \pm}$. Let $Z:=\left\{u \in X_{-}: A u \in Y_{+}\right\}$. If

$$
d:=\operatorname{dim}\left(Z / X_{+}\right)<\infty,
$$


then

$$
\text { ind } A_{-}-\text {ind } A_{+}=d \text {. }
$$

Proof. $\operatorname{ran} A_{+} \subset \operatorname{ran} A_{-}$, so $\operatorname{ker} A_{-}^{*}=\left(\operatorname{ran} A_{-}\right)^{\perp} \subset\left(\operatorname{ran} A_{+}\right)^{\perp}=\operatorname{ker} A_{+}^{*}$; both spaces are finite-dimensional by Fredholmness of $A_{ \pm}$. Choose a basis $\psi_{1}, \ldots, \psi_{r}$ for ker $A_{+}^{*} \subset Y_{+}^{*}$ so that $\psi_{m+1}, \ldots, \psi_{r}$ form a basis for ker $A_{-}^{*}$. Choose $w_{1}, \ldots, w_{r} \in Y_{+}$biorthogonal to $\psi_{1}, \ldots, \psi_{r}$. Then $w_{1}, \ldots, w_{m} \in\left(\operatorname{ker} A_{-}^{*}\right)^{\perp}=$ $\operatorname{ran} A_{-}$by choice of $m$, so we can find $u_{1}, \ldots, u_{m} \in X_{-}$with $A u_{j}=w_{j} \in Y_{+}$, which also means $u_{1}, \ldots, u_{m} \in Z$ by definition of $Z$.

Claim: $u_{1}, \ldots, u_{m}, \operatorname{ker} A_{-}$are independent modulo $X_{+}$. If not, we could find nontrivial coefficients $\alpha_{1}, \ldots, \alpha_{m}$ as well as $k \in \operatorname{ker} A_{-}, x \in X_{+}$, so that

$$
\sum_{i=1}^{m} \alpha_{i} u_{i}=k+x
$$

Then

$$
A \sum_{i=1}^{m} \alpha_{i} u_{i}=A k+A x=A x
$$

so

$$
\alpha_{j}=\psi_{j}\left(\sum_{i=1}^{m} \alpha_{i} w_{i}\right)=\psi_{j}\left(A \sum_{i=1}^{m} \alpha_{i} u_{i}\right)=\psi_{j}(A x)=0 \quad(j=1, \ldots, n)
$$

since $\psi_{j} \in \operatorname{ker} A_{+}^{*}=\left(\operatorname{ran} A_{+}\right)^{\perp}$ and $A x \in \operatorname{ran} A_{+}$. The coefficients are trivial - contradiction.

Assume we can add a $u_{m+1} \in Z$ so that $u_{1}, \ldots, u_{m+1}$, ker $A_{-}$are still independent modulo $X_{+}$. The system

$$
\psi_{j}\left(A \sum_{i=1}^{m+1} \alpha_{i} u_{i}\right)=0 \quad(j=1, \ldots, m)
$$

is underdetermined, so we can find a nontrivial solution $\alpha_{1}, \ldots, \alpha_{m+1}$. Combined with the same result for $j=m+1, \ldots, r$ (trivial) and with

$$
\psi\left(A \sum_{i=1}^{m+1} \alpha_{i} u_{i}\right)=0 \quad \text { for } \psi \in \operatorname{ker} A_{-}^{*} \subset \operatorname{ker} A_{+}^{*},
$$

we obtain

$$
\psi\left(A \sum_{i=1}^{m+1} \alpha_{i} u_{i}\right)=0 \quad \text { for all } \psi \in \operatorname{ker} A_{+}^{*},
$$

i.e.

$$
A \sum_{i=1}^{m+1} \alpha_{i} u_{i} \in \operatorname{ker}\left(A_{+}^{*}\right)^{\perp}=\operatorname{ran} A_{+},
$$


but then

$$
A\left(\sum_{i=1}^{m+1} \alpha_{i} u_{i}-d\right)=0
$$

for some $d \in X_{+}$, so $u_{1}, \ldots, u_{m+1}$, $\operatorname{ker} A_{-}$are dependent modulo $X_{+}-$contradiction.

Hence $u_{1}, \ldots, u_{m}$ form the basis of a complement of ker $A_{-}$in $Z$ modulo $X_{+}$, $\mathrm{sd}^{22}$

$$
\operatorname{dim}\left(Z / X_{+}\right)=\operatorname{dim}\left(\operatorname{ker} A_{-} / X_{+}\right)+m
$$

Finally,

$$
\begin{aligned}
& \text { ind } A_{-}-\operatorname{ind} A_{+} \\
& =\left(\operatorname{dim} \operatorname{ker} A_{-}-\operatorname{dim} \operatorname{ker} A_{-}^{*}\right)-\left(\operatorname{dim} \operatorname{ker} A_{+}-\operatorname{dim} \operatorname{ker} A_{+}^{*}\right) \\
& =\left(\operatorname{dim} \operatorname{ker} A_{-}-\operatorname{dim} \operatorname{ker} A_{+}\right)+\left(\operatorname{dim} \operatorname{ker} A_{+}^{*}-\operatorname{dim} \operatorname{ker} A_{-}^{*}\right) \\
& =\operatorname{dim}\left(\operatorname{ker} A_{-} / \operatorname{dim} \operatorname{ker} A_{+}\right)+m=\operatorname{dim}\left(\operatorname{ker} A_{-} / X_{+}\right)+m \\
& =\operatorname{dim}\left(\operatorname{ker} A_{-} / X_{+}\right)+\operatorname{dim}\left(Z / X_{+}\right)-\operatorname{dim}\left(\operatorname{ker} A_{-} / X_{+}\right)=\operatorname{dim}\left(Z / X_{+}\right) .
\end{aligned}
$$

\section{References}

[1] G. Ben-Dor. Shock Wave Reflection Phenomena. Springer, 1992.

[2] G. Ben-Dor. A state-of-the-knowledge review on pseudo-steady shock-wave reflections and their transition criteria. Shock Waves, 15:277-294, 2006.

[3] G. Ben-Dor, M. Ivanov, E.I. Vasilev, and T. Elperin. Hysteresis processes in the regular reflection $\leftrightarrow$ Mach reflection transition in steady flows. Progr. Aero. Sci., 38:347-387, 2002.

[4] Gui-Qiang Chen and M. Feldman. Global solutions to shock reflection by large-angle wedges for potential flow. Annals of Math. To appear.

[5] Gui-Qiang Chen, Yongqian Zhang, and Dianwen Zhu. Existence and stability of supersonic euler flows past Lipschitz wedges. Arch. Rat. Mech. Anal., 181(2):261-310, 2006.

[6] V. Elling. Nonuniqueness of entropy solutions and the carbuncle phenomenon. In Proceedings of the 10th Conference on Hyperbolic Problems (HYP2004), volume I, pages 375-382. Yokohama Publishers, 2005. http://www.umich.edu/ velling/hyp2004-colour.ps.

${ }^{22}$ We write $V / W:=V /(V \cap W)$ for simplicity. 
[7] V. Elling. A possible counterexample to well-posedness of entropy solution and to Godunov scheme convergence. Math. Comp., 75:1721-1733, 2006. See also arxiv:math.NA/0509331.

[8] V. Elling. Regular reflection in self-similar potential flow and the sonic criterion. Submitted. See also arxiv:0710.0104, 2007.

[9] V. Elling and Tai-Ping Liu. Physicality of weak Prandtl-Meyer reflection. In RIMS Kokyuroku, volume 1495, pages 112-117. Kyoto University, Research Institute for Mathematical Sciences, May 2006. http://www.umich.edu/ ${ }^{\sim}$ velling/rims05.ps.

[10] V. Elling and Tai-Ping Liu. Supersonic flow onto a solid wedge. Comm. Pure Appl. Math., 61(10):1347-1448, 2008.

[11] D. Gilbarg and N.S. Trudinger. Elliptic Partial Differential Equations of Second Order, volume 224 of A Series of Comprehensive Studies in Mathematics. Springer, 2nd edition, 1983.

[12] P. Grisvard. Elliptic Problems in Nonsmooth Domains. Pitman, 1985.

[13] K.G. Guderley. The Theory of Transonic Flow. Pergamon Press, Oxford, 1962.

[14] L.F. Henderson, K. Takayama, W.Y. Crutchfield, and S. Itabashi. The persistence of regular reflection during strong shock diffraction over rigid ramps. J. Fluid Mech., 431:273-296, 2000.

[15] H.G. Hornung. On the stability of steady-flow regular and mach reflection. Shock Waves, 7:123-125, 1997.

[16] J. Hunter and M. Brio. Weak shock reflection. J. Fluid Mech., 410:235-261, 2000 .

[17] J. Hunter and A. Tesdall. Self-similar solutions for weak shock reflection. SIAM J. Appl. Math., 63(1):42-61, 2002.

[18] P. Krehl and M. van der Geest. The discovery of the Mach reflection effect and its demonstration in an auditorium. Shock Waves, 1:3-15, 1991.

[19] C. De Lellis and L. Székelyhidi Jr. On admissibility criteria for weak solutions of the Euler equations. Technical report. Preprint arxiv:0712.3288v1 [math.AP].

[20] G. Lieberman. Oblique derivative problems in Lipschitz domains II. Discontinuous boundary data. J. reine angew. Math., 389:1-21, 1988.

[21] E. Mach and J. Wosyka. Über die Fortpflanzungsgeschwindigkeit von Explosionsschallwellen. Sitzungsber. Akad. Wiss. Wien (II. Abth.), 72:44-52, 1875 . 
[22] V. Maz'ya, S. Nazarov, and B. Plamenevskii. Asymptotic Theory of Elliptic Boundary Value Problems in Singularly Perturbed Domains, volume I. Birkhäuser, 2000.

[23] V.G. Maz'ya and B.A. Plamenevskii. Estimates in $L_{p}$ and in Hölder classes and the Miranda-Agmon maximum principle for solutions of elliptic boundary value problems in domains with singular points. Amer. Math. Soc. Transl. (2), 123:1-88, 1984.

[24] S.A. Nazarov and B.A. Plamenevskii. Elliptic Problems in Domains with Piecewise Smooth Boundaries. W. de Gruyter, 1994.

[25] J. von Neumann. Oblique reflection of shocks. Technical Report 12, Navy Dep., Bureau of Ordnance, Washington, D.C., 1943. In: Collected works, v. 6 , p. 238-299.

[26] B.W. Skews and J.T. Ashworth. The physical nature of weak shock wave reflection. J. Fluid Mech., 542:105-114, 2005.

[27] M. van Dyke. An Album of Fluid Motion. The Parabolic Press, Stanford, California, 1982.

[28] E.I. Vasilev and A.N. Kraiko. Numerical simulation of weak shock diffraction over a wedge under the von Neumann paradox conditions. Comp. Math. Math. Phys., 39(8):1335-1345, 1999.

[29] P. Woodward and P. Colella. The numerical simulation of two-dimensional fluid flow with strong shocks. J. Comp. Phys., 54:115-173, 1984. 